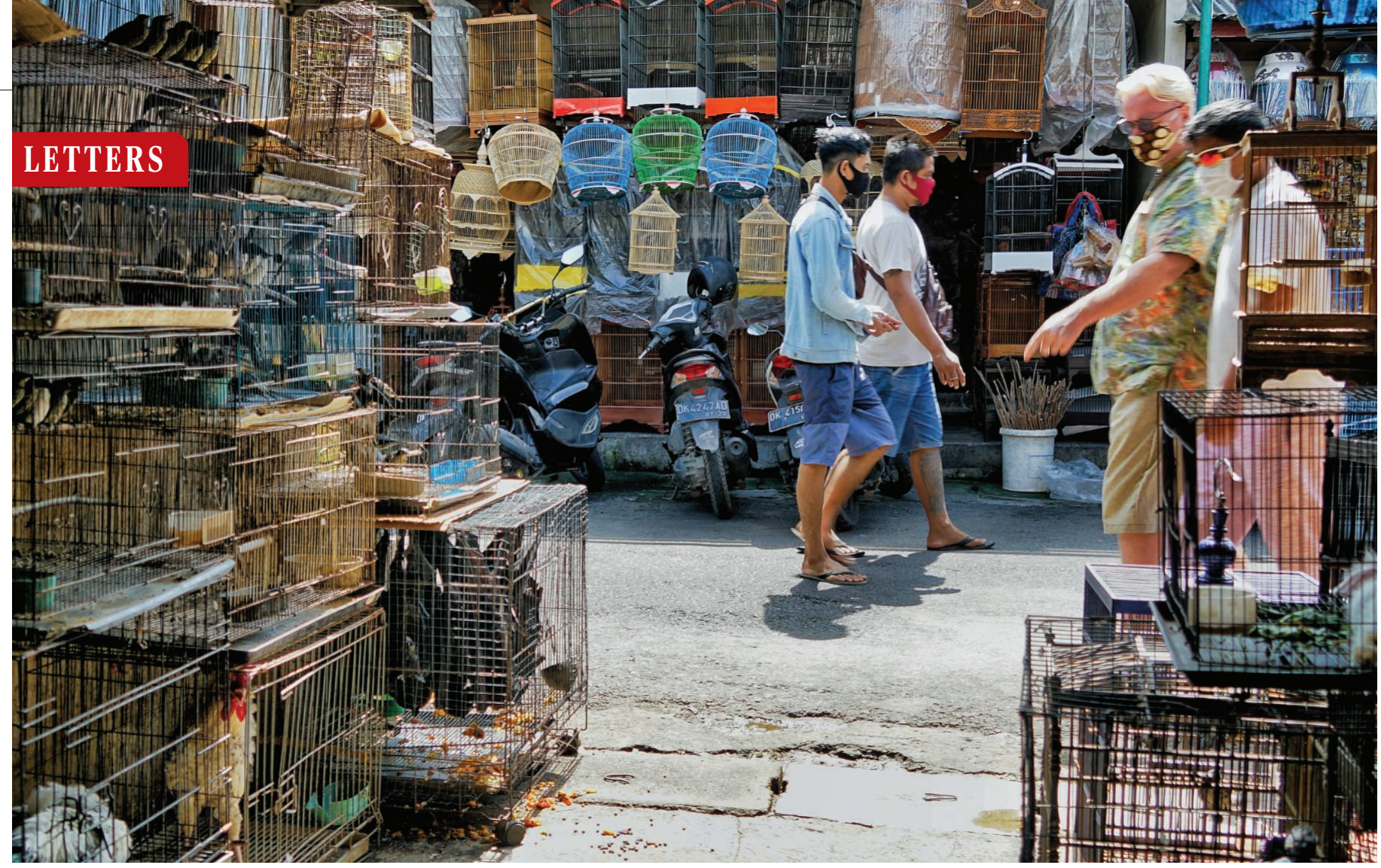

Disease transmission can occur at live animal markets, but zoonotic disease research could benefit from an emphasis on humans' and animals' shared risk of infection.

Edited by Jennifer Sills

\section{EcoHealth reframing of disease monitoring}

Decade-old (1) and recent warnings for coronaviruses with zoonotic epidemic potential (2) could have prevented the emergence of coronavirus disease 2019 (COVID-19) (3). We therefore agree with Watsa and colleagues ("Rigorous wildlife disease surveillance," Perspective, 10 July, p. 145) that wildlife biosurveillance should increase. However, representing animals as a threat to humans through disease transmission leads to ill-conceived reactive policies (4). A perspective (5) in which animals and humans share similar risks of pathogens and infections, making animals relevant disease models and sentinels, would be more effective. Clarifying the connection between animal and human health could increase public support for research seeking to understand host-switching in animals, such as the study of virus evolution (6), interactions in pathogen communities (7), and pathogen discovery (8).

A shared-risk perspective on emerging infectious diseases mirrors the field of EcoHealth, which explores the links between ecosystem, animal, and human health. Such strategies place value in healthy ecosystems through an integrative approach that considers both pathogen biodiversity and social-ecological drivers (9). Prevention based on understanding the transmission of pathogens through EcoHealth-based emerging infectious disease surveillance is a promising avenue for sustainability science, orders of magnitude cheaper than mitigation in response to a transfer to human hosts (10), and less intrusive than current crisis responses.

Maarten P.M. Vanhove1, ${ }^{1,23 *}$, Jean Hugé $e^{4,5,6}$, Luc Janssens de Bisthoven ${ }^{7}$, Hans Keune ${ }^{8,9}$, Anne Laudisoit $^{10}$, Séverine Thys ${ }^{11}$, Erik Verheyen ${ }^{12,13}$, Nicolas Antoine-Moussiaux ${ }^{14}$

${ }^{1}$ Research Group Zoology: Biodiversity and Toxicology, Centre for Environmental Sciences, Hasselt University, Diepenbeek. Belgium. ${ }^{2}$ Department of Botany and Zoology, Faculty of Science, Masaryk University, Brno, Czech Republic. ${ }^{3}$ Laboratory of Biodiversity and Evolutionary Genomics, Department of Biology, University of Leuven, Leuven, Belgium. ${ }^{4}$ Department of Environmental Science, Open University of the Netherlands, Heerlen, Netherlands. ${ }^{5}$ Department of Biology, Vrije Universiteit Brussel, Brussels, Belgium. ${ }^{6}$ Research Group Environmental Biology, Centre for Environmental Sciences, Hasselt University. Diepenbeek, Belgium. ${ }^{7}$ Capacities for Biodiversity and Sustainable Development, Royal Belgian Institute for Natural Sciences, CEBioS program, Brussels, Belgium. ${ }^{8}$ Belgian Biodiversity Platform-Research Institute Nature and Forest, Brussels, Belgium. ${ }^{9} \mathrm{Chair}$ Care and the Natural Living Environment, Department of Primary and Interdisciplinary Care Antwerp, Faculty of Medical and Health Sciences, University of Antwerp. Antwerp, Belgium. ${ }^{10}$ Ecohealth Alliance, New York, NY 10018, USA. ${ }^{11}$ Department of Vaccinology,
Faculty of Medicine and Health Sciences, University of Antwerp, Antwerp, Belgium. ${ }^{12}$ Operational Directorate Taxonomy and Phylogeny, Royal Belgian Institute for Natural Sciences, Brussels, Belgium. ${ }^{13}$ University of Antwerp, Department of Biology, Evolutionary Ecology, Antwerp, Belgium. ${ }^{14} \mathrm{~F} u n d a m e n t a l$ and Applied Research for Animals and Health, Faculty of Veterinary Medicine, University of Liège, 4000 Liège, Belgium.

*Corresponding author.

Email: maarten.vanhove@uhasselt.be

\section{REFERENCES AND NOTES}

1. V.C.C.Cheng, S. K. P. Lau, P.C.Y.Woo, K. Yung Yuen, Clin. Microbiol. Rev. 20,660 (2007)

2. Y. Fanetal. Viruses 11.210 (2019).

3. European Environment Agency (EEA), “Late lessons from early warnings: Science, precaution, innovationSummary" (Report1/2013, Publications Office of the European Union, Luxembourg, 2013); www.eea.europa. eu/publications/late-lessons-2.

4. N. Antoine-Moussiaux etal Sustain. Sci. 14,1729 (2019).

5. P. M. Rabinowitz, L. Odofin, F.J. Dein, EcoHealth 5 , 224 (2008).

6. S.J. Anthony etal. Virus Evol. 3, vex012 (2017).

7. W. de Souza, Parasitol. Res. 119, 2369 (2020).

8. D. R. Brooks et al., WCSA Journal 1,1(2020).

9. H. Lerner, C. Berg, Front. Vet. Sci. 4.163(2017).

10. A. P. Dobson et al., Science 369, 379 (2020).

10.1126/science.abe8239

\section{Build international biorepository capacity}

In their Perspective "Rigorous wildlife disease surveillance" (10 July, p. 145), M.

Watsa et al. underscore the value of One Health approaches to stimulate integration 
across currently siloed efforts in zoonotic research and mitigation. To achieve comprehensive decentralized pathogen surveillance, there is an urgent need to develop environmental and biodiversity infrastructure in biodiverse countries experiencing high rates of habitat conversion, wildlife trafficking, and human-wildlife interactions.

Approximately one-third of One Health networks lack an environmental component, fewer than half are active in wildlife surveillance, and almost none is led by developing countries (1). International support for development of natural history museums with frozen vertebrate tissue collections remains a key component missing from the One Health equation. Most pathogens causing severe outbreaks in humans are zoonotic in origin (2); thus, understanding their evolution and that of their wild animal hosts is imperative.

As was the case for coronavirus disease 2019 (COVID-19) (3), identifying wild animal reservoirs can be challenging when biorepositories are lacking (4). In most countries, natural history biorepositories remain poorly supported and largely disconnected from public health initiatives. For example, most studies of bat coronaviruses to date (5), including the PREDICT animal surveys discussed in Watsa et al., did not preserve host specimens or tissues, thus limiting the potential for molecular host identification or replication and extension of the science (6). Emerging infectious disease response hinges on sampling depth across space, time, and taxonomy, the very sampling enabled by museum biorepositories. As primary biological infrastructure, in-country development of museum collections that follow best practices (7), with specimen data freely available through the internet, should be an international imperative ( 8 ) for effective global surveillance and mitigation of emerging infectious diseases.

Jocelyn P. Colella', Bernard Risky Agwanda², Faisal Ali Anwarali Khan ${ }^{3}$, John Bates ${ }^{4,5}$, Carlos A. Carrión Bonilla ${ }^{6,7}$, Noé U. de la Sancha ${ }^{4,8}$, Jonathan L. Dunnum ${ }^{7}$, Adam W. Ferguson ${ }^{4}$, Stephen E. Greiman ${ }^{9}$, Prince Kaleme Kiswele ${ }^{10}$, Enrique P. Lessa ${ }^{11}$, Pamela Soltis ${ }^{12}$, Cody W. Thompson ${ }^{13}$, Maarten P. M. Vanhove ${ }^{14,15,16}$, Paul W. Webala ${ }^{17}$, Marcelo Weksler ${ }^{18}$, Joseph A. Cook ${ }^{7 *}$

'Biodiversity Institute, University of Kansas, Lawrence, KS 66045 USA. ${ }^{2}$ National Museums of Kenya, Nairobi, Kenya. ${ }^{3}$ Faculty of Resource Science and Technology. Universiti Malaysia Sarawak, Sarawak, Malaysia. ${ }^{4}$ Field Museum Chicago, IL 60605, USA. ${ }^{5}$ Natural Science Collections Alliance, Washington, DC 20005 , USA. ${ }^{6}$ Museo de Zoologiá, Escuela de Biología,
Pontificia Universidad Catolica del Ecuador, Quito Ecuador. ${ }^{7}$ Museum of Southwestern Biology and Biology Department, University of New Mexico, Albuquerque, NM 87131, USA. ${ }^{8}$ Department of Biological Sciences, Chicago State University, Chicago, IL 60628, USA. ' Department of Biology, Georgia Southern University, Statesboro, GA 30458, USA. ${ }^{10}$ Centre de Recherche en Sciences Naturelles, Lwiro, Democratic Republic of Congo. ${ }^{11}$ Facultad de Ciencias, Universidad de la República, Montevideo, Uruguay. ${ }^{12}$ Florida Museum of Natural History and the University of Florida Biodiversity Institute, University of Florida, Gainesville, FL 32611, USA. ${ }^{13}$ Department of Ecology and Evolutionary Biology and the Museum of Zoology, University of Michigan, Ann Arbor, MI 48108, USA. ${ }^{14}$ Research Group Zoology: Biodiversity and Toxicology, Centre for Environmental Sciences, Hasselt University, Diepenbeek, Belgium. ${ }^{15}$ Department of Botany and Zoology, Faculty of Science, Masaryk University, Brno, Czech Republic. ${ }^{16}$ Laboratory of Biodiversity and Evolutionary Genomics, Department of Biology, University of Leuven, Leuven, Belgium. ${ }^{17}$ Department of Forestry and Wildlife Management, Maasai Mara University, Narok, Kenya. ${ }^{18}$ Departamento de Vertebrados, Museu Nacional, Universidade Federal do Rio de Janeiro, Rio de Janeiro, Brazil.

*Corresponding author. Email: cookjose@unm.edu

\section{REFERENCES AND NOTES}

1. M. S. Khan et al., Lancet Planet. Health 2, e264 (2018).

2. K. E. Jones et al., Nature 451,990 (2008).

3. J. Cohen, Science 10.1126/science.abd7707 (2020).

4. S. A. J. Leendertz, J. F. Gogarten, A. Düx, S. CalvignacSpencer, F. H. Leendertz. EcoHealth 13,18 (2016).

5. B. Huet al.,PLoS Path.13, e1006698 (2017).

6. J.A. Cooket al., Bioscience 70,531(2020).

7. J.L. Dunnumet al.,PLoS Negl. Trop. Dis. 11,1(2017)

8. O. Paknia, H. Sh Rajaei, A. Koch, Organ. Divers. Evol. 15 619 (2015).

10.1126/science.abe4813

\section{Response}

We agree with Vanhove $e t$ al. that wildlife conservation and emerging infectious disease screening are two sides of the same coin. Wildlife and humans can be vulnerable to spillover events by the same pathogen. For example, respiratory diseases (1) and Ebola virus (2) outbreaks have occurred simultaneously in great apes and humans. Pathogens also affect biogeographical species range expansions, contractions, and extinctions (3). Biosurveillance efforts should reflect that health risks are shared by humans and wildlife, a central tenet of the One Health framework (4). As Vanhove et al. point out, wildlife can serve as the source for preventive solutions that mitigate spillover risks into humans and animals.

A shared risk perspective could also combat the narratives that portray animals as dangerous pests or disposable commodities that endanger human health (5), as in the case of bats (6), many of which are likely not hosts for coronaviruses such as severe acute respiratory syndrome coronavirus 2 (SARS-CoV-2) (7). In addition to emphasizing shared risk, such misinformation can be countered with well-researched messaging following a zoonotic outbreak. Conservation social science has honed a suite of tools to identify the often unpredictable human motivations behind (8), and the possible negative consequences of, such communications (9).

Colella et al. suggest that surveillance efforts should include natural history collections. Some natural history museums and zoos archive biobanked specimens, cryopreserved viable cell cultures, disease specimen banks, and histopathology samples, but this highly effective practice (10) is limited by high costs. We agree that devoting funding toward biodiversity banking within countries at high risk for emerging infectious diseases would improve conservation outcomes. Taxonomically diverse biobanked tissues and live cell cultures could expand studies of host-pathogen relationships, clarifying host range or affected tissues and providing in vitro systems for infectivity and pathogenicity investigations. Such collections could allow drug development for humans to expand beyond just a few animal laboratory models, given that relatively well-studied viruses such as SARS-CoV-2 are potentially broadly infectious across taxonomic orders (11). Comparative genomics and transcriptomics among nonmodel species are used infrequently in biomedical research programs but hold great potential for prioritizing species and gene targets with alternative host defense mechanisms for laboratory study (12).

\section{Mrinalini Watsa ${ }^{1,2 *}$ and Wildlife Disease} Surveillance Focus Group ${ }^{3}$

'Population Sustainability, San Diego Zoo Global, San Diego, CA 92027, USA. ${ }^{2}$ Field Projects International, San Diego, CA 92126, USA. ${ }^{3}$ Wildlife Disease Surveillance Focus Group authors and affiliations are listed at science.sciencemag.org/ content/369/6500/145/suppl/DC1.

*Corresponding author.

Email: merkenswickwatsa@sandiegozoo.org

\section{REFERENCES AND NOTES}

1. J.D. Negrey et al., Emerg. Microbes Infect. 8,139 (2019).

2. S.A.J. Leendertzet al., Mamm. Rev.47, 98 (2017).

3. R. E. Ricklefs, E. Bermingham, Glob. Ecol. Biogeogr.11, 353 (2002).

4. P. M. Rabinowitz et al., Ecohealth 5,224 (2008).

5. J.P. Kibambe et al., "In Africa, wildlife raises the risk of deadly diseases: It doesn't have to" CNN (2020).

6. H.Zhao, Science 367, 1436 (2020).

7. H. Yanetal., biorxiv 10.1101/2020.09.08.284737 (2020).

8. K. E. Wallen, E. Daut, Nat. Conserv. 26,55 (2018)

9. H. N. Dang Vu, M. R. Nielsen, Hum. Dimensions Wildl.23, 417 (2020)

10. J. Radin, J. Cult. Econ. 8,361(2015).

11. J. Damas et al., Proc. Natl. Acad. Sci. U.S.A.117, 22311 (2020).

12. L. Yurkovetskiy et al., Cell 183,739 (2020). 


\section{Science}

\section{Build international biorepository capacity}

Jocelyn P. Colella, Bernard Risky Agwanda, Faisal Ali Anwarali Khan, John Bates, Carlos A. Carrión Bonilla, Noé U. de la Sancha, Jonathan L. Dunnum, Adam W. Ferguson, Stephen E. Greiman, Prince Kaleme Kiswele, Enrique P. Lessa, Pamela Soltis, Cody W. Thompson, Maarten P. M. Vanhove, Paul W. Webala, Marcelo Weksler and Joseph A. Cook

Science 370 (6518), 773-774.

DOI: $10.1126 /$ science.abe4813

ARTICLE TOOLS

http://science.sciencemag.org/content/370/6518/773.2

RELATED

http://science.sciencemag.org/content/sci/370/6518/773.1.full

http://science sciencemag.org/content/sci/370/6518/774. full

http://science.sciencemag.org/content/sci/369/6500/145.full

REFERENCES

This article cites 7 articles, 0 of which you can access for free http://science.sciencemag.org/content/370/6518/773.2\#BIBL

PERMISSIONS

http://www.sciencemag.org/help/reprints-and-permissions

Use of this article is subject to the Terms of Service

Science (print ISSN 0036-8075; online ISSN 1095-9203) is published by the American Association for the Advancement of Science, 1200 New York Avenue NW, Washington, DC 20005. The title Science is a registered trademark of AAAS.

Copyright @ 2020 The Authors, some rights reserved; exclusive licensee American Association for the Advancement of Science. No claim to original U.S. Government Works 